\title{
Ultrasonic Exposure of the Liquid Film for Highly Efficient Gas Absorption
}

\author{
VLADIMIR N. KHMELEV, ROMAN N. GOLYKH, SERGEY N. TSYGANOK, \\ GALINA A. BOBROVA \\ Chair of Methods and Tools of Measurement and Automation \\ Biysk Technological Institute (branch) of Altai State Technical University named after I.I. Polzunov \\ 659305, Altai region, Biysk city, street named after Hero of Soviet Union Trofimov, 27
}

\section{RUSSIAN FEDERATION}

\begin{abstract}
A model of ultrasonic intensification of the absorption process is proposed, developed, and analyzed. For the first time, the model takes into account the effect of wave-like capillary perturbations of the liquid-gas surface and the acceleration of diffusion in the liquid volume on the absorption rate due to cavitation. According to the results of the model researches, the need for uniform sounding of the extended surface of the liquid film is established to accelerate the absorption of carbon dioxide and other harmful and target gaseous impurities by at least 3 times. The designs and layout of ultrasonic vibration radiators with an extended radiation surface are proposed. The results of measurements of the vibration amplitudes of the invented transducers showed that they have a relative deviation of the amplitude of no more than 0.2. The proposed approaches to the implementation of the process can be recommended for further research, the selection of optimal designs, and industrial applications to accelerate the absorption of gaseous impurities.
\end{abstract}

Key-Words: - Ultrasonic, absorption, liquid film, gas, wave-guide

Received: February 7, 2021. Revised: August 23, 2021. Accepted: September 3, 2021. Published: September 8, 2021.

\section{Introduction}

Today, the reduction of the total "carbon footprint" is an actual task of state policy of many countries. For this purpose, ecological programs are adopted, energy-saving production is encouraged and motivated, and the philosophy of consumption is changing. According to forecasts, the world economy will spend more than $\$ 1$ trillion on reducing carbon emissions in the next 10 years [1].

Behind the words about the struggle for the preservation of the climate and the environment, there are obvious economic interests. Goods and services in countries with a low "carbon footprint" will gain additional competitive advantages.

The natural absorption of carbon dioxide by forests and other natural ecosystems does not cope with the high "carbon footprint" in the atmosphere, which has accumulated rapidly in recent decades as a result of industrialization.

The artificial methods of carbon dioxide absorption developed to help nature, implemented in various devices, have limited capabilities and today do not even partially solve the problem.

At the same time, the problem of gas absorption and separation is relevant not only for the removal of carbon dioxide from the atmosphere. Also, absorption of other explosive, flammable and toxic impurities (methane, propane, butane, carbon monoxide, hydrogen sulfide, radon, mercury vapor, etc.) from the gas emissions of technological processes is relevant. Moreover, individual gas components can serve as valuable raw materials (for example, hydrogen sulfide for the production of sulfuric acid). The release of certain gases is necessary for targeted use as energy carriers (for example, pure hydrogen) for engineering and biomedical applications.

The existing attempts to create and use industrial devices lead either to a decrease in the quality of the resulting gas product (in terms of purity) or to a disproportionate increase in energy consumption, which makes the process of separating gas mixtures economically unprofitable.

The analysis of scientific, technical, and patent literature carried out to find ways to develop gas separation devices allowed us to establish that the existing methods of gas separation, depending on the underlying physical principle, are divided into three types:

1) cryogenic methods (based on the boiling point difference of the mixes components), which are described in patents US20100251765, JP2008196806. Cryogenic methods are the most energy-intensive because of the need to cool the gas 
mixture to the boiling point of the lowest boiling component. Therefore, these methods are unsuitable for highly efficient separation of gaseous mixtures that contain components with a boiling point close to absolute zero (for example, helium - -268.93 and hydrogen - -252.8 degrees Celsius). In addition, gas separation plants based on cryogenic methods are characterized by the complexity of the design. Plus, this physical principle of gas separation is characterized by a long starting period necessary for cooling the gas mixture to its boiling point;

2) membrane methods (based on the difference in the penetration rates of the components of the gas mix through the membrane substance), which are described in patents US10092880, US8092581, US9808772, US10913027, RU2035981, RU2347604. Membrane methods also have a high energy consumption due to the need for compressed air supply. Along with this, the gases obtained in existing membrane devices, as a rule, have a low degree of purity (for example, no more than $50 \%$ when obtaining oxygen). Attempts to increase the degree of purity by increasing the pressure of compressed air require a disproportionate increase in energy consumption, which makes membrane methods economically unprofitable;

3) sorption methods (based on the difference in the rate of absorption of the components of the mix by a liquid or solid sorbent), which are described in patents US9868085, US20070256559, US20140090556， US20020162454， RU2474462, RU2464071.

Sorption methods consist in the selective absorption of a component (sorbent) of a gas mixture by a sorbent-a solid or a liquid. According to the nature of the absorption of the sorbent, sorption methods are divided into two types:

- adsorption - concentration of the sorbent on the interface of the phases or its absorption by the surface layer of the sorbent;

- absorption - volumetric absorption, in which the sorbent is distributed over the entire volume of the sorbent (or absorbent).

If at the same time the formation of chemical bonds of the component of the gas mixture with the substance of the sorbent occurs, then such methods are also called chemisorption.

Among the sorption methods, absorption is of the greatest interest, since it allows for the absorption of the gas component by the entire volume of the sorbent, unlike adsorption, in which the component is concentrated only on the surface.

However, due to the multiple increasing in the need for gas separation, today this productivity is not enough.
Therefore, one of the possible ways to increase the absorption performance is the use of external energy influences that contribute to an increase in the liquid-gas interface and the diffusion coefficient.

More than 60 years ago, it was proposed to use ultrasonic exposure on the liquid film [2,3]. The obtained experimental results indicate the effectiveness of such exposure and an increase in the productivity in various variants of the interaction of the gas flow with the fluctuating liquid (up to $10 \ldots 15 \%$ when the gas and liquid flows move in parallel and up to $30 \%$ and more when it is difficult to implement bubbling) $[2,3,4,5]$.

The absorption efficiency is increasing due to ultrasonic cavitation, which accelerates of liquid saturation by gas and increases "liquid-gas" interface.

However, the positive effects that occur when ultrasonic vibrations are applied for cavitationacoustic acceleration of absorption processes have been obtained exclusively in laboratory conditions to date, without achieving practical use.

This is due to the following reasons:

- insufficient knowledge of the physical mechanisms of absorption intensification in the cavitation-acoustic field;

- as a result, there is no scientific basis for optimizing energy dosing (determining modes) and conditions for entering ultrasonic vibrations;

- the absence of ultrasonic radiators that allow effective ultrasonic effects on thin films of liquids with a large spreading area (more than $0.3 \mathrm{~m}^{\wedge} 2$ ).

Over the past years, no qualified researches, which have solved these problems and conducted to identify the optimal modes conditions for the formation of liquid vibrations for effective interaction with the gas flow.

The many publications about related topics exist in last 2 years [6-9].

Therefore, the first stage of research is the creation of a model of ultrasonic absorption intensification to identify the optimal modes (in terms of frequency and intensity of vibrations) and conditions (in terms of the area of the voiced surface) of ultrasonic exposure.

The proposed and developed model and its analysis are presented below.

\section{Problem Formulation}

Ultrasonic absorption intensification is implemented in a two-layer "gas-liquid" system, as shown in Fig. 1. 


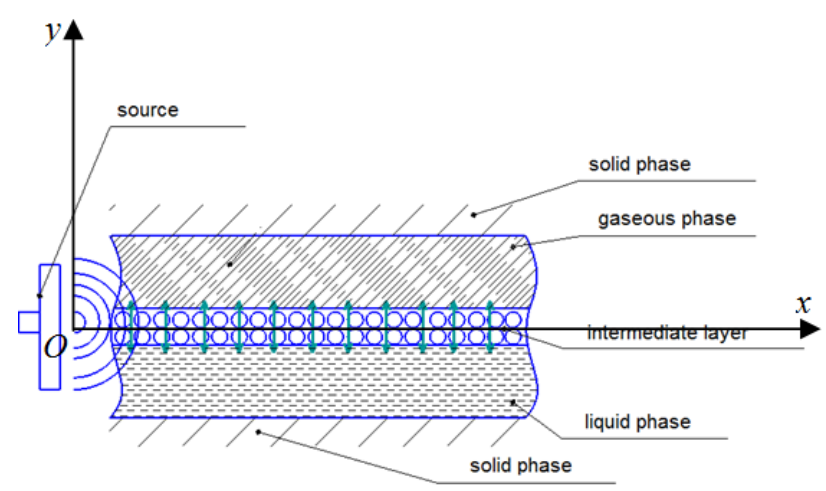

Fig. 1: Two-layer "gas-liquid" system for ultrasonic absorption intensification

The liquid phase covers the solid surface in the form of a thin film. Between the liquid and the gas, there is an intermediate layer. This is a transition layer, which contains the concentration gradient of the liquid and gas molecules. The transition layer can be subjected to wave-like perturbations under ultrasonic vibration exposure. This will increase the liquid-gas interface [10-12].

At the same time, it is known that ultrasonic exposure not only forms wave-like disturbances on the liquid-gas surface but also accelerates the processes of mass transfer of diffusion in the volume of the liquid phase [13].

In this regard, the simulation of absorption should take into account the ultrasonic acceleration of both stages of the process-absorption through the intermediate layer and transport in the volume of the gas phase.

In general, the mathematical formulation of the problem of modeling the absorption process is formulated as follows (the process is considered stationary):

$$
\begin{aligned}
& \left(\mathbf{u}_{g}, \nabla\right) C_{g}=D_{g} \Delta C_{g} \\
& \left(\mathbf{u}_{l}, \nabla\right) C_{l}=D_{l} \Delta C_{l} \\
& \left.D_{l} \frac{\partial C_{l}}{\partial y}\right|_{y=h}=\left.D_{g} \frac{\partial C_{g}}{\partial y}\right|_{y=h}=-\left.K_{S}\left(C_{l}-C_{g}\right)\right|_{y=h}
\end{aligned}
$$

where $C_{l}$ is the concentration of the target component in the liquid phase, $\mathrm{kg} / \mathrm{m}^{3} ; D_{l}$ is the average diffusion coefficient in the liquid phase, $\mathrm{m}^{2} / \mathrm{s} ; \mathbf{u}_{l}$ is the flow rate of the liquid phase, $\mathrm{m} / \mathrm{s} ; C_{g}$ is the concentration of the target component in the gas phase, $\mathrm{kg} / \mathrm{m}^{3} ; D_{g}$ is the mass transfer coefficient in the gas phase, $\mathrm{m}^{2} / \mathrm{s} ; \mathbf{u}_{g}$ is the flow rate of the liquid phase, $\mathrm{m} / \mathrm{s} ; K_{S}$ is the concentration interaction coefficient, $\mathrm{m} / \mathrm{s} ; h$ is the thickness of the liquid film, $\mathrm{m}$.

Standard analytical and numerical methods for solving equations (1-3) are known [14-18]. However, the submodels are needed to calculate the kinetics and efficiency of the absorption process intensified by ultrasonic vibrations. These submodels determining the equivalent averaged coefficients in equations (1-3).

When creating submodels, the following assumptions are made:

1) The instantaneous diffusion processes of the target component inside the bubble compared to the diffusion in the liquid phase (a sharp jump in the concentration of the target component), since the diffusion proceeds faster in gases.

Based on the reference data, the volume diffusion coefficient $\left(D_{g}\right)$ of carbon dioxide in the air is $0.14 \cdot 10^{-4} \mathrm{~m}^{2} / \mathrm{s}$ without ultrasound [19]. The volume diffusion coefficient $\left(D_{l}\right)$ of carbon dioxide in water is $0.000015 \cdot 10^{-4} \mathrm{~m}^{2} / \mathrm{s}$ [20]. This suggests that the diffusion of the target component in the liquid phase is much slower than in the gas phase $\left(D_{l} \ll D_{g}\right)$. Therefore, it is sufficient to determine only one (the diffusion coefficient in the liquid) from the two averaged diffusion coefficients (under the ultrasonic exposure).

2) The absorbent liquid is linear-viscous (Newtonian). The non-Newtonian media are usually high-molecular (highly dispersed) and not used for gas absorption.

3) The number of cavitation bubbles in the liquid is determined by the processes of their coalescence during expansion and crushing during their collapse.

\subsection{Submodel for Determining the Equivalent Diffusion Coefficient in the Liquid Phase}

The equivalent diffusion coefficient in the liquid phase is primarily determined by cavitation processes.

The main parameters determining diffusion coefficient is bubble radius and concentration of cavitation bubbles.

The dependence of the bubble radius from the time in the expansion stage is described by the Nolting-Neppiras equation (4) in the compression stage equation Kirkwood-Bethe (5) based on the assumption that the fluid is linear-viscous:

$$
\begin{aligned}
& \rho\left(\frac{3}{2}\left(\frac{\partial R}{\partial t}\right)^{2}+R \frac{\partial^{2} R}{\partial t^{2}}\right)=-4 \mu \frac{\frac{\partial R}{\partial t}}{R}+ \\
& +p_{v}+\left(p_{0}+\frac{2 \sigma}{R_{0}}\right)\left(\frac{R_{0}}{R}\right)^{3 \gamma}-p_{\infty}
\end{aligned}
$$




$$
\begin{aligned}
& R \frac{\partial^{2} R}{\partial t^{2}}\left(1-\frac{\frac{\partial R}{\partial t}}{C}\right)+\frac{3}{2}\left(\frac{\partial R}{\partial t}\right)^{2}\left(1-\frac{\frac{\partial R}{\partial t}}{3 C}\right)= \\
& =H\left(1+\frac{\frac{\partial R}{\partial t}}{C}\right)+\frac{\partial H}{\partial t} \frac{R}{C}\left(1-\frac{\frac{\partial R}{\partial t}}{C}\right)
\end{aligned}
$$

where $R$ is the instantaneous bubble radius, m; $\rho$ is density of cavitation medium, $\mathrm{kg} / \mathrm{m}^{3} ; p_{0}$ is the static pressure in the fluid, $\mathrm{Pa} ; \mu$ is the liquid viscosity, $\mathrm{Pa} \cdot \mathrm{s} ; \sigma$ is the surface tension of the liquid phase, $\mathrm{N} / \mathrm{m} ; \mathrm{H}$ is the enthalpy of the liquid, $\mathrm{m}^{2} / \mathrm{s}^{2} ; C$ is the local speed of sound in the liquid phase, $\mathrm{m} / \mathrm{s}$.

The determination of the concentration of cavitation bubbles was carried out in a small region $\Omega$, the size of which is much smaller than the length of the ultrasonic wave and much larger than the size of an individual cavitation bubble. The area is schematically shown in Fig. 2.

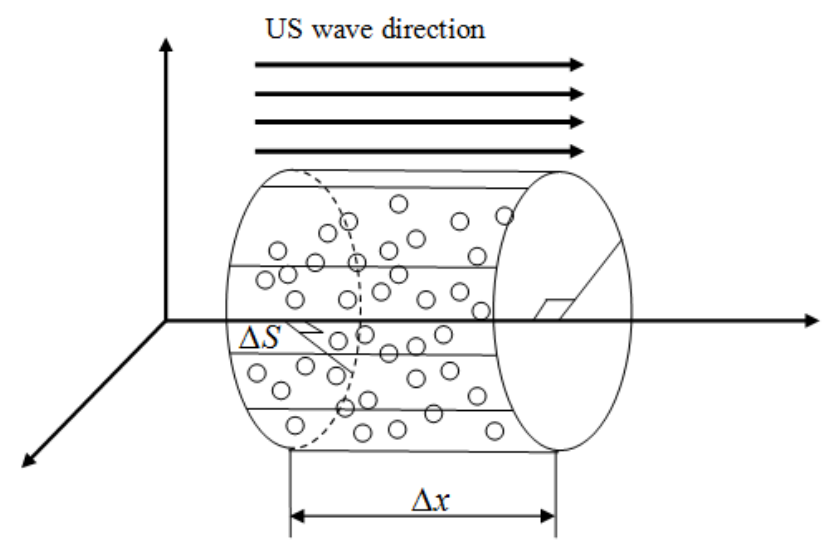

Fig. 2: Schematic representation of a local region of a liquid with cavitation bubbles

The concentration of cavitation bubbles is determining by two processes, which are coalescence of bubbles during each bubble expansion and breakup into multiple nuclei during collapse. The concentration is determined by equation (6) [21]:

$$
\frac{\partial n}{\partial t}=\frac{n(j-1)}{i T_{0}}-k_{B} n^{2}
$$

where $n$ is the number concentration of cavitation bubbles as a function of time $t, \mathrm{~m}^{-3} ; i$ is average number of bubbles pulsations up to its collapse; $k_{B}$ is constant of coalescence speed of bubbles, $\mathrm{m}^{3} / \mathrm{s} ; T_{0}$ is period of ultrasonic oscillations, $\mathrm{s} ; j$ average number of nuclei formed by separate bubble breakup.

The stationary concentration, when coalescence and breakup processes are in equilibrium, is independent on initial concentration of cavitation nuclei and determined by following expression:

$$
n_{b u b}=\frac{j-1}{i k_{B} T_{0}} \text {. }
$$

The coalescence constant is proportional to average velocity (over ultrasonic oscillation period) of bubbles convergence and bubbles collision crosssection:

$$
k_{B}=\frac{S_{e f f}\langle u\rangle}{2} ;
$$

where $S_{\text {eff }}$ is effective collision cross-section of bubbles (it is determined by distance between bubbles, at this the coalescence duration is much less than ultrasonic oscillations period), $\mathrm{m}^{2} ;\langle u\rangle$ is average speed of bubbles convergence, $\mathrm{m} / \mathrm{s}$.

$S_{\text {eff }}$ is determined by:

$S_{\text {eff }}=25 \pi R_{M A X}{ }^{2}$;

The average bubbles convergence speed is determined by equation obtained by authors from 2nd I. Newton's law taking into account Bjerknes forces action:

$$
\begin{aligned}
& \frac{4 \pi R_{0}{ }^{3}}{3} \rho_{G} \frac{\partial^{2} \mathbf{d}_{12}}{\partial t^{2}}= \\
& =-2 \frac{4 \pi R^{3}}{3\left|\mathbf{d}_{12}\right|^{3}} \rho_{L} \frac{\partial\left(R^{2} \frac{\partial R}{\partial t}\right)}{\partial t} \mathbf{d}_{12}+ \\
& +\frac{1}{2} \frac{\partial}{\partial t}\left(\frac{4 \pi R^{3}}{3} \rho_{L}\left(-\frac{\partial \mathbf{d}_{12}}{\partial t}\right)\right)+ \\
& +4 \pi \eta R\left(-\frac{\partial \mathbf{d}_{12}}{\partial t}\right)
\end{aligned}
$$

where $\mathbf{d}_{12}$ is $3 \mathrm{D}$ vector of distance between two bubbles, $\mathrm{m} ; R_{0}$ is initial radius of bubble, $\mathrm{m} ; \rho_{\mathrm{G}}$ is density of gaseous phase is nuclei, $\mathrm{kg} / \mathrm{m}^{3} ; \rho_{\mathrm{L}}$ is density of liquid phase, $\mathrm{kg} / \mathrm{m}^{3} ; \eta$ is viscosity of liquid, $\mathrm{Pa} \cdot \mathrm{s}$.

The numerical solution of equation (10) made it possible to reveal the dependence of the vector of the line of the centers of two separate bubbles on time and calculate the average speed of their convergence according to the following expression:

$$
\langle u\rangle=\frac{\left|\mathbf{d}_{12}(T)-\mathbf{d}_{12}(0)\right|}{T} .
$$

The number of nuclei $j$ after breakup was calculated by indirect method using experimental data about concentration bubbles dependency on ultrasonic intensity [22]. 
At known bubbles concentration and radius the bubble volume content was determined:

$$
\delta_{l}(t)=\frac{4}{3} \pi R^{3}(t) n_{b u b}=\frac{4(j-1) \pi R^{3}(t)}{3 i k_{B} T_{0}} .
$$

Further, the expression for bubble volume content is using for determination equivalent diffusion coefficient and increasing of interface "gas-liquid".

The cavitating liquid is heterogeneous medium. Thus, for determining diffusion coefficient the microscopic diffusion equation (that takes into account the difference between the coefficient in the solid-liquid phase and in the cavitation cavity) is used:

$$
\frac{\partial C}{\partial t}+(\mathbf{u}, \nabla) C=\operatorname{div}\left(D_{\text {micro }}(\mathbf{r}) \nabla C\right)
$$

where $D_{\text {micro }}$ is the microscopic diffusion coefficient, $\mathrm{m}^{2} / \mathrm{s}$.

In the equation, the diffusion coefficient depends on the spatial coordinate and is determined by the type of phase at the observed point (gas phase, liquid phase, or transition layer between phases). It is a continuously differentiable function concerning the coordinate due to the presence of a transition layer. Therefore, it is necessary to average this equation over a volume (whose dimensions are much smaller than the thickness of the liquid film, but much larger than the cavitation bubble) to calculate the absorption capacity.

According to generalization of researches and estimations, the acoustic streaming weak influence on diffusion coefficient [23-25].

The determination of the equivalent diffusion coefficient for the gas-liquid mix by averaging equation (11) by volume was carried out using result of researches presented in $[20,26]$. According to these researches, the diffusion coefficient for a liquid containing gas bubbles is:

$$
D=\frac{1-y_{A}}{\frac{y_{B}}{D_{l 0}}+\frac{y_{C}}{D_{g 0}}} ;
$$

where $D$ is the averaged diffusion coefficient, $\mathrm{m}^{2} / \mathrm{s}$; $D_{l 0}, D_{g 0}$ is the diffusion coefficient in the liquid and gas phases, $\mathrm{m}^{2} / \mathrm{s} ; y_{A}, y_{B}, y_{C}$ is volume concentrations of absorbed gas in different phases.

Taking into account the accepted assumption 1) $\left(\frac{y_{C}}{D_{g 0}}<<\frac{y_{B}}{D_{l 0}} ;\right.$ the volume diffusion coefficient $\left(D_{g}\right)$ of carbon dioxide in the air is $0.14 \cdot 10^{-4} \mathrm{~m}^{2} / \mathrm{s}$ without ultrasound [19]; the volume diffusion coefficient $\left(D_{l}\right)$ of carbon dioxide in water is $0.000015 \cdot 10^{-4}$ $\mathrm{m}^{2} / \mathrm{s}$ [20]) that the expression is converted to the following simplified form:

$$
D=\frac{D_{l 0}}{1-\delta_{l}}
$$

where $\delta_{l}$ is the volume content of the bubbles (cavitation index).

This diffusion coefficient is averaged over the vibration period (14) since the volume content $\delta_{l}$ of the bubbles depends on time (due to the expansion and collapse of the bubbles).

$$
\langle D\rangle=\frac{1}{T} \int_{0}^{T} \frac{D_{l 0} d t}{1-\delta_{l}(t)} ;
$$

where $T$ is period of ultrasonic oscillations, s.

\subsection{Submodel for Determining the Equivalent Coefficient of Concentration Interaction in the Liquid Phase}

Since the concentration interaction coefficient is proportional to the interfacial surface area

$$
K_{S}=K_{S 0} \frac{S_{U S}}{S_{\text {NoUS }}} ;
$$

is necessary to study the formation of capillary waves affecting the area.

The model developed by the authors for determining the relative increase in the area of the interfacial surface $\frac{S_{U S}}{S_{\text {No US }}}$ is based on the equations of each stage of the formation of capillary waves: the spherically symmetric expansion of the cavitation bubble; its asymmetric collapse; the formation of narrowly directed shock waves and the formation of wave-like perturbations of the gasliquid interface profile.

When considering the expansion stage of a cavitation bubble, its maximum radius $R_{M A X}$ is determined based on the Nolting-Neppiras equation (4). Then the shape of the bubble is determined when it collapses from the maximum radius $R_{M A X}$ to the minimum size based on the integral equation (16) with boundary conditions for the potential of the liquid velocity on the bubble wall (17).

$$
\begin{aligned}
& \frac{\varphi\left(\mathbf{r}_{0}\right)}{2}=\int_{S_{A} \cup S_{B}}\left(E_{\mathbf{r}_{0}} V_{\mathbf{n}}-\frac{\partial E_{\mathbf{r}_{0}}}{\partial \mathbf{n}} \varphi\right) \partial S ; \\
& \frac{\partial \varphi}{\partial t}+\frac{\left|V_{\mathbf{n}}\right|^{2}+\left|V_{\boldsymbol{\tau}}\right|^{2}}{2}= \\
& =\frac{2 \sigma K}{\rho}-\frac{p_{v}}{\rho}\left(\frac{3 V}{4 \pi R_{M A X}{ }^{3}}\right)^{\gamma} ;
\end{aligned}
$$

where $\mathbf{r}_{0}, \mathbf{r}$ are the coordinate vectors of the points of the wall of the cavitation bubble or the surface "gas-liquid", $\mathrm{m} ; \varphi$ is the potential of the velocity of 
the liquid on the wall of the cavitation bubble $S_{A}$ or the solid surface $S_{B}, \mathrm{~m}^{2} / \mathrm{s} ; V_{\mathbf{n}}$ and $V_{\tau}$ are the normal and tangential components of the velocity of the liquid, $\mathrm{m} / \mathrm{s} ; E_{r_{0}}(\mathbf{r})$ is the fundamental solution of the Laplace equation; $V$ is the volume of the cavitation bubble, $\mathrm{m}^{3} ; p_{\mathbf{n}}$ is the pressure of saturated vapors of the liquid; $\rho$ and $\sigma$ are the density $\left(\mathrm{kg} / \mathrm{m}^{3}\right)$ and surface tension $(\mathrm{N} / \mathrm{m})$ of the liquid, respectively; $K$ is the average the curvature of the bubble walls, $\mathrm{m}^{-1}$.

Since the bubble is a hemispherical radiator of the shock wave, the pressure profile which is on the border of the "liquid-gas" received approximated by equation (18):

$$
\begin{aligned}
& p(\mathbf{r}, t)=\sum_{n=-\infty}^{\infty} f a \varepsilon \operatorname{Re} \int_{0}^{\frac{\pi}{2}} \int_{0}^{\frac{2 \pi}{\omega}} p_{c}\left(t_{1}\right) \times \\
& \times e^{\frac{2 \pi i f n}{c}\left(c\left(t-t_{1}\right)+\frac{a}{\varepsilon}-\frac{\eta n \pi f}{\rho c^{2}}+z \varepsilon \cos \psi\right)} \times \quad ; \\
& \times \sin \psi J_{0}\left(2 \pi n \frac{f}{c} r \varepsilon \sin \psi\right) \partial t_{1} \partial \psi \\
& \varepsilon=\frac{a}{\sqrt{r^{2}+z^{2}}}
\end{aligned}
$$

where $(r, z)$ - coordinates of a point of the boundary "liquid-gas", $\mathrm{m} ; f$ is frequency of ultrasonic exposure, $\mathrm{s}^{-1} ; t$ and $t_{1}$ is the time points, $\mathrm{s} ; \eta$ is the viscosity of the liquid, $\mathrm{Pa} \cdot \mathrm{s} ; \rho$ and $\mathrm{c}$ are the density $(\mathrm{kg} / \mathrm{m} 3)$ and the speed of sound $(\mathrm{m} / \mathrm{s})$ in the liquid, respectively; $p_{c}$ the pressure in the core of a cavitation bubble, $\mathrm{Pa} ; a$ is the radius of the cavitation bubble when it reaches the high pressure at its core, $\mathrm{m}$.

The found shock wave pressure profile is used in the future to determine the relative increase in the surface area of the phase interface based on the obtained expression (19), which takes into account the change in the concentration of bubbles due to their coalescence and crushing:

$$
\begin{aligned}
& \frac{S_{U S}}{S_{\text {NoUS }}}=1+ \\
& +\frac{4 h}{\pi} f\left(\frac{j-1}{R_{M A X}{ }^{2} U} \int_{0}^{0,5 \lambda} r \sqrt{1+\left(\frac{1}{\rho} \int_{0}^{t} \int_{0}^{t_{2}} \int_{0}^{\partial^{2} p} \frac{\partial^{2}}{\partial z \partial r} \partial t_{1} \partial t_{2}\right)^{2}} d r\right. \\
& \int_{0}^{t} \int_{0}^{t_{2}} \frac{\partial^{2} p}{\partial z \partial r}\left(\frac{\lambda}{2}, z, t_{1}\right) \partial t_{1} \partial t_{2}=0
\end{aligned}
$$

where $\lambda$ is the wavelength of capillary waves $(\mathrm{m})$ determined by the condition (15); $R_{M A X}$ is the maximum bubble radius, $\mathrm{m}$; $U$ is the average speed of convergence of the bubbles, $\mathrm{m} / \mathrm{s} ; j$ is the number of nuclei formed during the fragmentation of the individual vial; $<>$ is the sign of averaging over the thickness of the liquid film; $h$ is the film thickness, m.

Thus, the proposed model of the process, the developed submodels, and their analysis allow us to identify all the necessary averaged coefficients (diffusion in the volume of the liquid phase and concentration interaction on the liquid-gas surface) to determine the efficiency of the absorption process.

The submodel for determining diffusion coeffient was presented in subsection 2.1 (see final expression (14)), and the model for determining concentration interaction coefficient (increasing the square of interface "liquid-gas") was presented in subsection 2.2 (see final expression (19)).

The data on ultrasonic exposure of vibration parameters (frequency and intensity) on the efficiency of the absorption process is presented below.

\section{Dependences of the Averaged Diffusion Coefficients and Concentration Interaction Determining the Absorption Efficiency}

The calculated dependencies of the equivalent diffusion coefficient based on the invented model are presented in Fig. 3a-f.

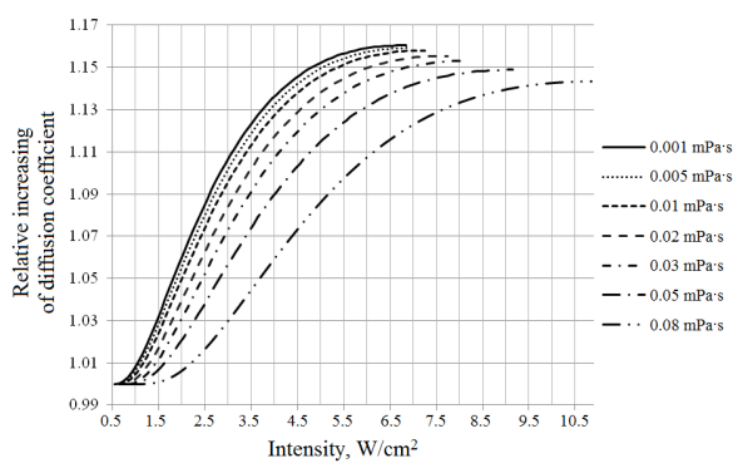

a) $22 \mathrm{kHz}$

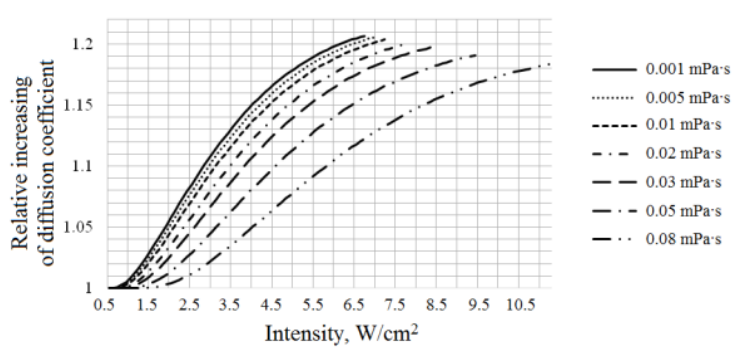

b) $25 \mathrm{kHz}$ 


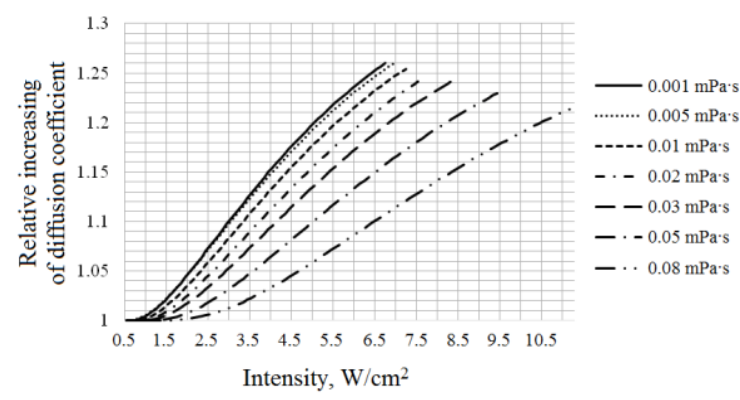

c) $30 \mathrm{kHz}$

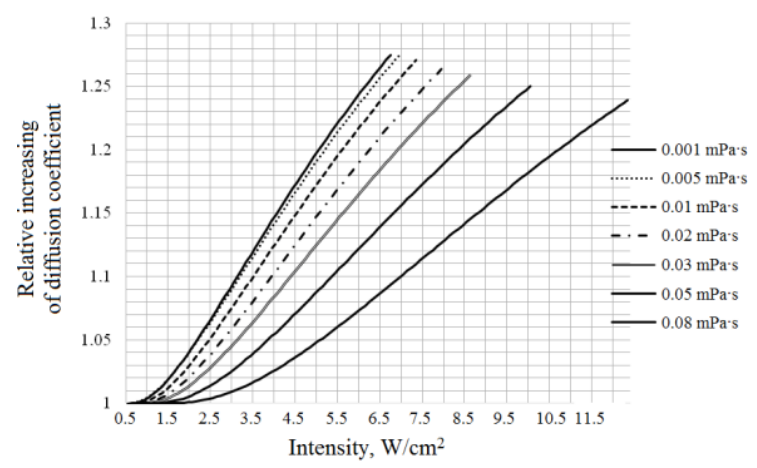

d) $33 \mathrm{kHz}$

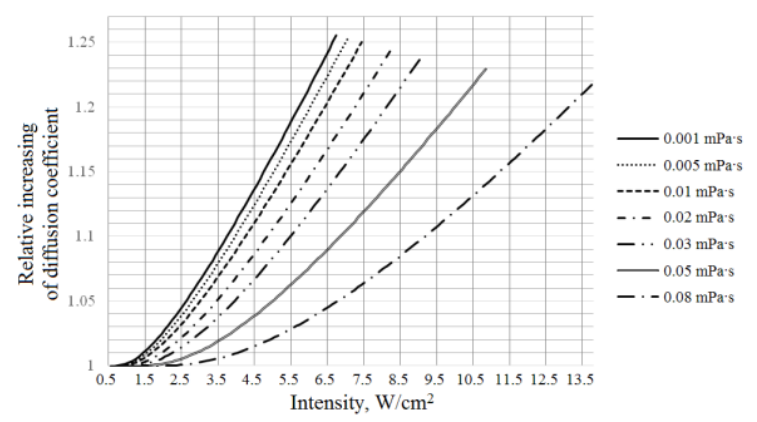

f) $44 \mathrm{kHz}$

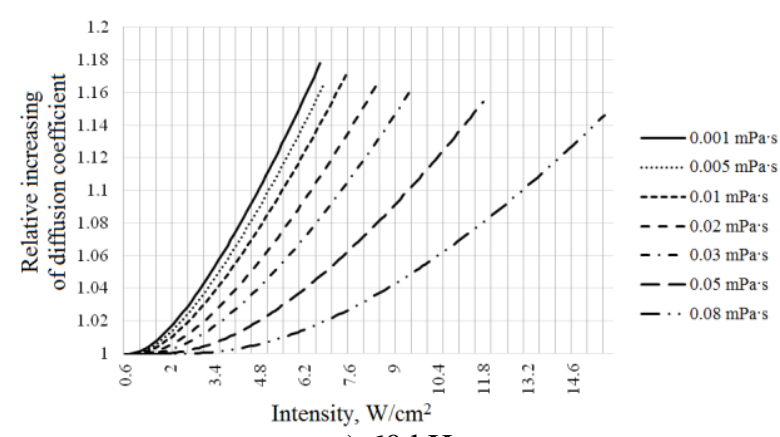

e) $60 \mathrm{kHz}$

Fig. 3: Dependences of the relative increases of the diffusion coefficient on the intensity of the vibrations at different viscosity (in $\mathrm{mPa} \cdot \mathrm{s}$ ) and frequency (in $\mathrm{kHz}$ )

As follows from the presented figures, the volume diffusion coefficient increases due to an increase in the cavitation index at increasing intensity.

The breakage of the graphs corresponds to the degenerate cavitation mode when the bubbles stop collapsing and degenerate into long-lived ones [27]. But, the breakage corresponds to the maximum achieved diffusion coefficient at a given vibration frequency and fluid properties due to the monotonous increase in the cavitation index with intensity increasing.

The analysis of the dependence of the maximum achieved diffusion coefficient on the frequency allowed us to establish that there is an optimal frequency of ultrasonic exposure at which the volume diffusion coefficient is maximum. The optimal frequency is $33 \mathrm{kHz}$. It does not depend on the viscosity of the absorbent liquid and the intensity of the vibrations.

A smaller number of larger cavitation bubbles are formed at frequencies below the optimal one.

Smaller bubbles are formed at frequencies above the optimal one and, accordingly, the critical radius of the bubble at which it degenerates into a longlived one also decreases. This determines the presence of the optimal frequency.

The diffusion is accelerated by up to $30 \%$ at the optimal frequency of exposure, the maximum permissible vibration intensity (at which the bubbles begin to degenerate into long-lived ones), and uniform sounding throughout the entire volume of the liquid. This is indicating the possibility of a significant increase in efficiency in comparison with the previously achieved experimental results.

It is necessary to investigate another factor that affects the efficiency of absorption (an increase in the surface area of the liquid-gas interface i.e. the equivalent coefficient of concentration interaction) for further investigation of the technology of gas absorption using ultrasonic exposure.

The calculated dependences of the equivalent coefficient of concentration interaction as a function of the amplitude of surface vibrations are shown in Fig. 4 and 5.

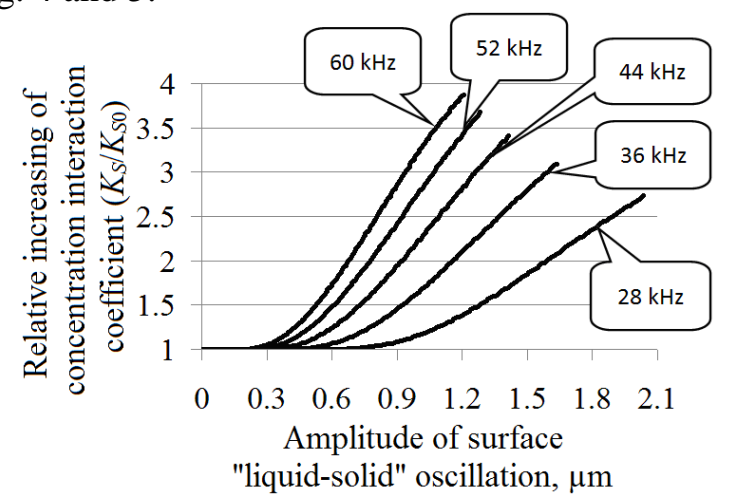

Fig. 4: Dependences of the relative increase in the concentration interaction coefficient on the amplitude of the vibrations at different frequencies 
The breakage of the graphs indicates that the capillary wave loses stability and begins to break up into droplets.

Fig. 5 shows the dependences of the specific surface area of the phase interface on the properties of the liquid (viscosity (a) and surface tension (b)), which have a significant effect on the profile of the phase contact surface along with the modes of ultrasonic exposure.

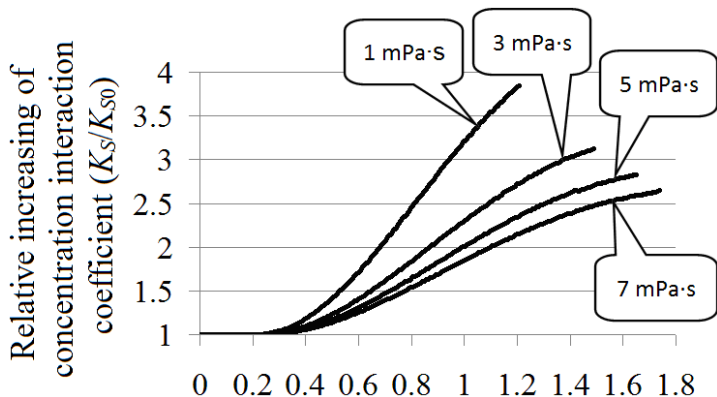

Amplitude of surface "liquid-solid" oscillation, $\mu \mathrm{m}$

a)

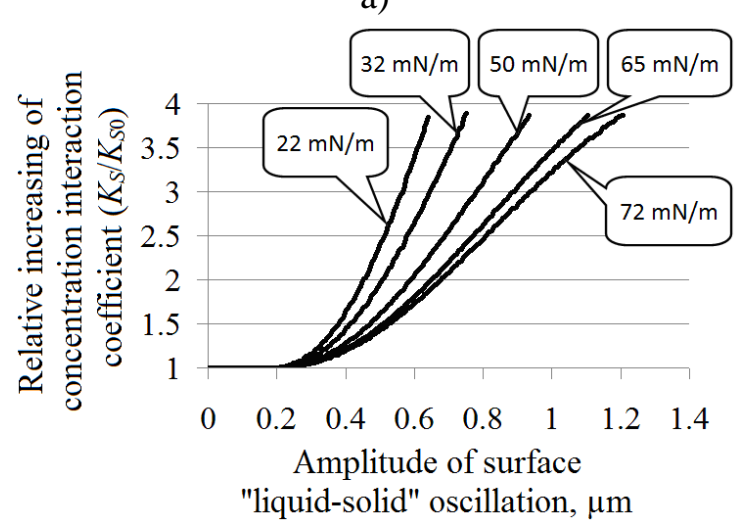

b)

Fig. 5: Dependence of the specific area of the phase interface on the vibration amplitude for different properties of the liquid (frequency $60 \mathrm{kHz}$ )

The dependencies shown in Fig. 5 allow us to conclude that an increase in viscosity leads to a decrease in the interface area due to the forces of viscous friction. At the same time, a decrease in the surface tension leads to an increase in the area, since the surface energy is directly related to it.

In the end, ultrasonic exposure allows you to increase the surface area of the liquid-gas interface by $2.5 . . .4$ times in a wide range of liquid properties.

Thus, ultrasonic exposure can theoretically accelerate the absorption process by $3 . . .5 .3$ times taking into account the increase in the diffusion rate in the liquid volume.

Our researches were shown that the increase in the efficiency of ultrasonic exposure on the gas absorption process is determined by the size of the interfacial interaction surface.

This requires the creation of special ultrasonic radiators with an extended vibrating surface. Our proposals for the creation and use of one radiator suitable for solving this problem are presented in the following section.

\section{Sonotrode with an Extended Radiation Surface}

The creation of ultrasonic vibrations must be carried out using an ultrasonic vibration system. The standard design contains a piezoelectric transducer, a booster (to increase the amplitude of the piezoelectric element), and an ultrasonic horn.

Now, the most common design of a vibratory system is a design with a cylindrical booster having a radial profile of longitudinal section [28-30].

It is necessary to attach several such transducers to the vibrating solid surface (an area much larger than $1 \mathrm{~m}^{2}$ ) as shown in Fig. 6 because the area of the output end of the transducer is small (no more than $8 \mathrm{~cm}^{2}$ ).

The wave of bending solid surface vibrations attenuate according to law $\frac{1}{\sqrt{k r}}$ (vibrations field from single radiator placed on infinite solid surface $w(r)=\frac{a}{J_{0}(k R)-\frac{J_{0}{ }^{\prime}(k R)}{Y_{0}{ }^{\prime}(k R)} Y_{0}(k R)}\left(J_{0}(k r)-\frac{J_{0}{ }^{\prime}(k R)}{Y_{0}{ }^{\prime}(k R)} Y_{0}(k r)\right) ;$ where $w$ is amplitude of oscillations of surface, $\mathrm{m} ; a$ is amplitude of oscillations of radiator, $\mathrm{m} ; r$ is distance from center of radiator, $\mathrm{m} ; R$ is radius of radiator, $\mathrm{m} ; k$ is wave number of surface vibrations, $\left.\mathrm{m}^{-1}\right)$.

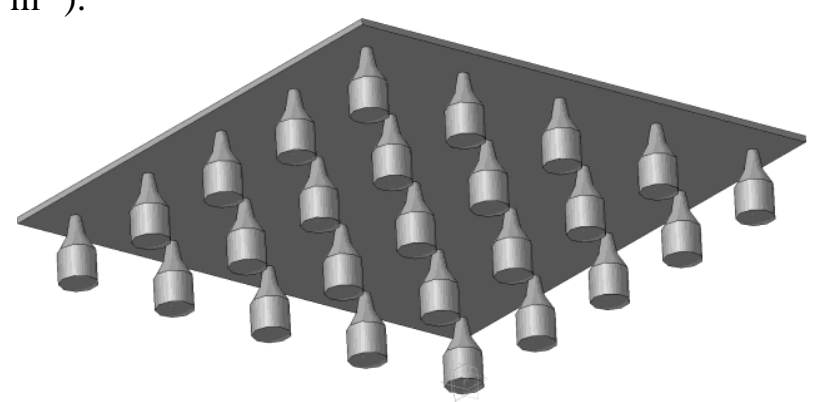

Fig. 6: Arrangement of cylindrical transducers for sounding a solid surface covered with a liquid film

Another condition for the uniform sounding of a solid surface is the absence of a phase difference in the vibrations of the output ends of different transducers. Unfortunately, it is impossible to provide the same vibration phases due to the inaccuracy of manufacturing, heterogeneity of the 
transducer's materials, and the piezoelectric elements. It was decided to develop transducers that have an expanded surface of the output end (flat sonotrodes). This, at least, helps to eliminate the influence of non-uniformity of piezoelectric element vibration.

The arrangement of transducers with an extended radiation surface is shown in Fig. 7.

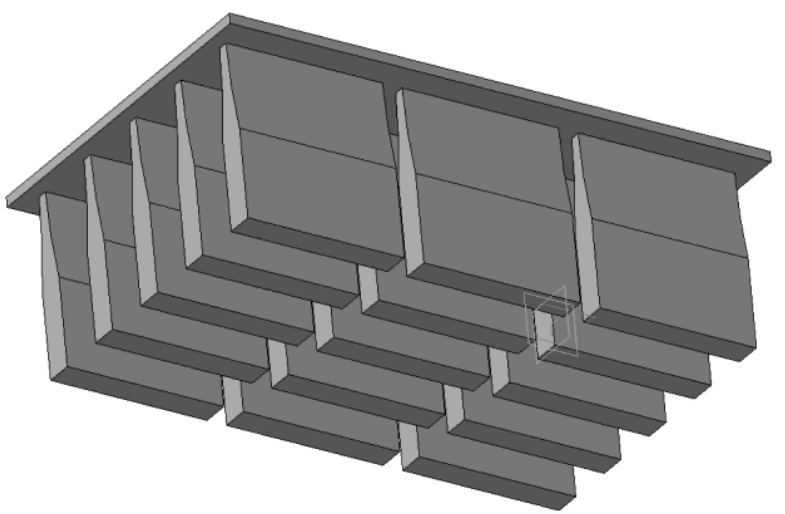

Fig. 7: Arrangement of transducers with an extended radiation surface for sounding a solid surface covered with a liquid film

The arrangement provides more uniform vibrations field. The fact is explained by following relations from theory of plates vibration:

$$
\begin{aligned}
& \frac{d^{4} w}{d x^{4}}-k^{4} w=0 ; \\
& w(0)=w(L)=a ; \\
& \frac{d w}{d x}(0)=\frac{d w}{d x}(L) ;
\end{aligned}
$$

where $\mathrm{x}$ is coordinate in axis, which is parallel to surface and perpendicular to radiator, $\mathrm{m} ; L$ is distance between neighbor radiators, $\mathrm{m} ; A$ is amplitude of oscillations of radiator, $\mathrm{m}$.

The solution of system (21-23) is following:

$$
\begin{aligned}
& w=A \sin (k x)+B \cos (k x)+C e^{k x}+D e^{-k x} ; \\
& A \sin (k L)+B \cos (k L)+C e^{k L}+D e^{-k L}=a ; \\
& B+C+D=a ; \\
& A+C-D=0 ; \\
& A \cos (k L)-B \sin (k L)+C e^{k L}-D e^{-k L}=0 . \\
& \left(\begin{array}{cccc}
\sin (k L) & \cos (k L) & e^{k L} & e^{-k L} \\
\cos (k L) & -\sin (k L) & e^{k L} & e^{-k L} \\
1 & 0 & 1 & -1 \\
0 & 1 & 1 & 1
\end{array}\right)\left(\begin{array}{c}
A \\
B \\
C \\
D
\end{array}\right)=a\left(\begin{array}{l}
1 \\
0 \\
0 \\
1
\end{array}\right) ;
\end{aligned}
$$

The acoustic field from the radiators has nonuniformity basically along $x$ axis. Coefficients $A$ and $B$ are non-zero. Thus, the field almost doesn't attenuate, but has form of standing wave.
Further, researches aimed at optimizing the size of such transducers are carried out. The optimizing is necessary to provide uniform vibrations of working end of separate transducer.

The researches were carried out by experimental methods (experimental samples of a transducer with an expanded radiation surface were made). The researches included measuring the distribution of the vibration amplitudes over the entire width of the transducer surface and identifying the width at which the relative deviation of the amplitudes is minimal.

The measurements were carried out at 9 points located at the same distance from each other, as shown in Fig. 8.

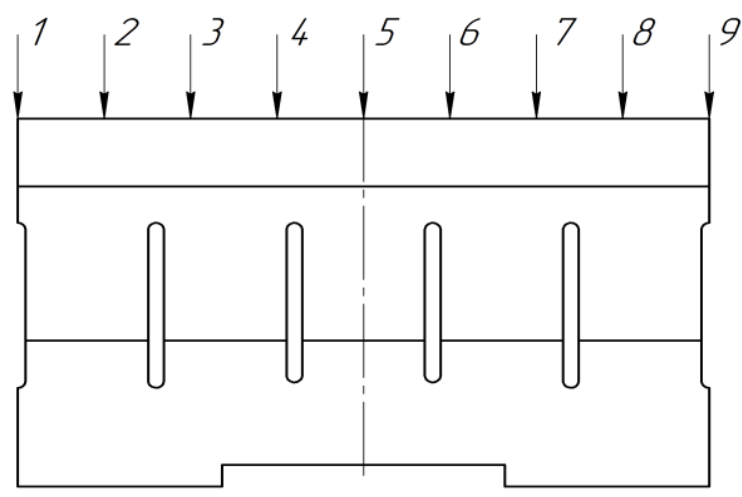

Fig.8: Selection of measurement points for the vibration amplitudes of transducers with an extended radiation surface

The vibration amplitudes $x$ were measured by the stroboscopic method. The measurement results are presented in Table 1.

Table 1. Measurement results obtained by the stroboscopic method

\begin{tabular}{|c|c|c|c|c|c|c|c|c|c|}
\hline $\begin{array}{c}\text { Horn } \\
\text { width, } \\
\text { mm }\end{array}$ & \multicolumn{6}{|c|}{ Peak-to-peak amplitude (double } \\
\cline { 2 - 9 } & 1 & 2 & 3 & 4 & 5 & 6 & 7 & 8 & 9 \\
\hline 150 & 23 & 23 & 32.5 & 33.5 & 37 & 33.5 & 32.5 & 23 & 23 \\
\hline 220 & 21 & 22.5 & 31.5 & 32,5 & 35 & 32.5 & 31.5 & 22.5 & 21 \\
\hline 270 & 24.5 & 24.5 & 35 & 36.5 & 39 & 36.5 & 35 & 24.5 & 24.5 \\
\hline
\end{tabular}

The relative deviations of the vibration ranges are calculated based on the obtained measurement results. 
Table 2. Relative deviations of the measurement results of the peak-to-peak amplitude (the line at the top means averaging over the area of the radiating

\begin{tabular}{|c|c|}
\hline surfac & $\frac{\sqrt{(x-\bar{x})^{2}}}{\bar{x}}$ \\
\hline $\begin{array}{c}\text { Horn width, } \\
\mathrm{mm}\end{array}$ & Relative deviation \\
\hline 150 & 0,1899 \\
\hline 220 & 0,1979 \\
\hline 270 & 0,1933 \\
\hline
\end{tabular}

The relative deviation of the amplitude in the entire range of horn widths does not exceed 0.2 as follows from the presented Table 2. While the calculations of the relative deviation for the disk radiator [23] showed that it is at least 0.7 (if the amplitude is approximated by a function $\left.A \frac{1+\cos (K r)}{2}\right)$.

The relative deviations of the amplitudes of flat transducers vary slightly in the width range from 150 to $270 \mathrm{~mm}$. According to the measurements, the maximum absolute values of the amplitude at a width of $270 \mathrm{~mm}$ no less than at a width of $150 \mathrm{~mm}$.

This indicates the feasibility of manufacturing transducers with an expanded radiation surface (width is $270 \mathrm{~mm}$ ) to intensify the absorption process.

\section{Conclusion}

A model of ultrasonic intensification of the absorption process is proposed, developed, and analyzed. For the first time, the model takes into account the effect of wave-like capillary perturbations of the liquid-gas surface and the acceleration of diffusion in the liquid volume on the absorption rate due to cavitation.

According to the results of the model researches, the need for uniform sounding of the extended surface of the liquid film is established to accelerate the absorption of carbon dioxide and other harmful and target gaseous impurities by at least 3 times.

The designs and layout of ultrasonic vibration radiators with an extended radiation surface are proposed. The results of measurements of the vibration amplitudes of the invented transducers showed that they have a relative deviation of the amplitude of no more than 0.2.

The proposed approaches to the implementation of the process can be recommended for further research, the selection of optimal designs, and industrial applications to accelerate the absorption of gaseous impurities.

The further developing of approaches and proposed model can be in following directions:

1. Creation of resonant conditions for crushing cavitation bubbles near the surface of the flowing liquid film and mechanical "capture" of the absorbed gas into the cavity of the deformed and destroyed bubble.

2. Crushing of bubbling inclusions due to cavitation in the liquid volume.

3. Construction of lattice Boltzmann equations $[25,26]$ using the apparatus of the theory of generalized functions that allow taking into account the impulse loading in the form of infinitely differentiable functions of volume forces that depend on the parameters of the random process of collapse of cavitation bubbles. The constructed lattice Boltzmann equations should allow us to calculate the crushing of cavitation bubbles near the surface of the flowing liquid film and the crushing of bubbling bubbles in the liquid volume due to cavitation.

4. Creation of an experimental installation for the study of the gas absorption process based on the proposed physical principles in conditions close to industrial ones.

The researches in the directions allow to solve the global problem of removal carbon footprint from atmosphere and high efficiency industrial gas separation.

\section{References:}

[1] (2016, January) The carbon footprint is the main threat of the 21st century, Komsomolskaya Pravda [Online], January, 2016. Available: https://www.irk.kp.ru/daily/26484/3354493/. In Russian.

[2] N. N. Maltsev, Absorption of benzene and the possibility of its intensification under the action of ultrasound: Ph. D. thesis, Dnepropetrovsk, UA, 1956.

[3] V.M. Ramm, Gas absorption, Moscow, RU: Chemistry, 1976.

[4] N. Khan, and Y. Adewuyi, Modeling the Ultrasonic Cavitation-Enhanced Removal of Nitric Oxide from Flue Gases In a Bubble Column Reactor, in American Institute of Chemical Engineerings 2008 Annual Meeting, 2008.

[5] F. Laugier, C. Andriantsiferana, A.M. Wilhelm, and H. Delmas, Ultrasound in gas-liquid systems: Effects on solubility and mass 
transfer, Ultrasonics Sonochemistry, Vol. 15, Iss. 6, 2008, pp. 965-972.

[6] S. Upreti, and A. Mehrotra, Experimental Determination of Gas Diffusivity in Liquids A Review, The Canadian Journal of Chemical Engineering, 99, 2020.

[7] E. Peleg, and A. Feigel, Turbulence and capillary waves on bubbles, Physical Review E, 104, 2021.

[8] G. Zhou, and A. Prosperetti, Capillary waves on a falling film, Physical Review Fluids, 5, 2020.

[9] S. Dyachenko, Traveling capillary waves on the boundary of a fluid disc, Studies in Applied Mathematics, August, 2021.

[10] A. Rahimzadeh, M.-R. Ahmadian-Yazdi, and M. Eslamian, Experimental study on the characteristics of capillary surface waves on a liquid film on an ultrasonically vibrated substrate, Fluid Dynamics Research, Vol. 50, No. 6, October 2018.

[11] A. Sugondo, Sutrisno, W. Anggono, and O. Anne, Effect of Frequency on Droplet Characteristics in Ultrasonic Atomization Process, in 2018 The 1st International Conference on Automotive, Manufacturing, and Mechanical Engineering (IC-AMME 2018), 2008, 7 p.

[12] V. Preobrazhensky, V. Aleshin, and P. Pernod, Explosive Instability of Gravity-Capillary Waves under Ultrasound Radiation Pressure, Phys. Wave Phen., 26, 2018, pp. 234-242.

[13] Y.G. Adewuyi, and N.E. Khan, Enhanced Removal of Nitrogen Oxide from Flue Gases in a Bubble Column Reactor, American Institute of Chemical Engineers Journal, Vol. 58, No. 8, 2012, pp. 2387-2411.

[14] Q. Xu, and H. An, A class of domain decomposition based nonlinear explicitimplicit iteration algorithms for solving diffusion equations with discontinuous coefficient, Journal of Computational and Applied Mathematics, 386, 2021.

[15] F. Muhiddin, and J. Sulaiman, Fourth-Order Crank-Nicolson Solution for Solving Diffusion Equation Using MSOR Iteration, Advanced Science Letters, 24, 2018, pp. 1912-1916.

[16] H. Huang, X. Wang, and X. Ma, A comparison study of solving diffusion equations with different algorithm methods, AIP Advances, 6, 2016.

[17] H. Jafari, H. Tajadodi, and S. Johnston, A decomposition method for solving diffusion equations via local fractional time derivative, Thermal Science, 19, 2015, pp. 123-129.
[18] M. Paripour, E. Babolian, and J. Saeidian, Analytic solutions to diffusion equations, Mathematical and Computer Modeling, 51, 2010, pp. 649-657.

[19] N.N. Koshkin, and M.G. Shirkevich, Elementary Physics Handbook, Moscow, RU: Science, 1976. In Russian.

[20] A.G. Novoselov, A.B. Duzhiy, and E.Y. Golikova, Molecular diffusion of gases in a liquid. Molecular diffusion coefficients of carbon dioxide in water, Scientific Journal of NRU IFMO. Series "Processes and devices of food productions”, No. 2, 2014. In Russian

[21] M.A. Margulis, and I.M. Margulis, Dynamics of bubbles ensemble in cavitation liquid, Journal of physical chemistry, 81, 12, 2007, pp. 2290-2295. In Russian.

[22] L.D. Rozenberg, Powerful ultrasonic fields, RU, Moscow: Science, 1968.

[23] V.N. Khmelev, A.V. Shalunov, R.N. Golykh, and V.A. Nesterov, Theoretical study coagulation of aerosols in thin resonant gaps, International Conference of Young Specialists on Micro/Nanotechnologies and Electron Devices, EDM'2019, Novosibirsk, Russia, 2019, pp. 180-187.

[24] O.V. Rudenko, and S.I. Soluyan, Theoretical foundations of nonlinear acoustics, Moscow, RU: Science, 1975. In Russian.

[25] K.S. Yong, and K. Sangmo, Acoustic Streaming, Encyclopedia of Microfluidics and Nanofluidics, 2015, pp. 1-15.

[26] C. Y. Wang, and C. Beckermann, A Multiphase Solute Diffusion Model for Dendritic Alloy Solidification, Metallurgical transactions A, Vol. 24A, 1993, pp. 2787-2802.

[27] R. Golykh, A. Shalunov, V. Khmelev, R. Lopatin, V. Minakov, and V. Shakura, Evaluation of Optimum Model and Conditions Providing Increasing Ultrasonic Cavitation Area in High-Viscous and Non-Newtonian Fluids, Romanian Journal of Acoustics and Vibration, Vol. 17, No. 2, 2020, pp. 101-108.

[28] I. Rosca, S. Chiriacescu, and N. Creţu, Ultrasonic horns optimization, Physics Procedia, 3, 2010, pp. 1033-1040.

[29] S.S. Khmelev, V.N. Khmelev, R.N. Golykh, and A.V. Shalunov, Development and research of concentrator-sonotrode with increased radiating surface, Archives of Acoustics, Vol. 40, No. 1, 2015, pp. 129-135.

[30] R. Paschke, Ultrasonic tool, patent US8961176B2, February 2015.

[31] O. Ilyin, Boundary conditions for lattice Boltzmann equations in applications to hemodynamics, Computer Research and Modeling, 12, 2020, pp. 865-882.

[32] A. Shore, The Lattice Boltzmann equation, Contemporary Physics, 59, 2019, pp. 1-1. 
Contribution of Individual Authors to the Creation of a Scientific Article (Ghostwriting Policy)

Vladimir N. Khmelev is author of conception, general coordinator of the research.

Roman N. Golykh has developed model of ultrasonic intensification of absorption.

Sergey N. Tsyganok has developed and ensured the manufactor of sonotrodes (wave-guides) with extended radiation surface.

Galina A. Bobrova has implemented program for numerial analysis of model, performed measurement of sonotrodes oscillations amplitudes.

Sources of Funding for Research Presented in a Scientific Article or Scientific Article Itself

The development of transducers with an extended radiation surface, the method of arrangement and the research of their functional capabilities were carried out with the financial support of the RFBR in the scientific project 20-21-00017 Rosatom.

Creative Commons Attribution License 4.0 (Attribution 4.0 International, CC BY 4.0)

This article is published under the terms of the Creative Commons Attribution License 4.0

https://creativecommons.org/licenses/by/4.0/deed.en _US 